\title{
Génomique de I'hémophilie de la reine Victoria
}

\author{
Simone Gilgenkrantz
}

Dans la nuit du 16 au 17 juillet 1918, le tsar Nicolas II, sa femme et ses 5 enfants, ainsi que trois domestiques et le médecin personnel du petit Alexis, atteint d'hémophilie, étaient massacrés à lekaterinbourg. II fallut presque 100 ans pour authentifier les restes retrouvés dans la forêt voisine de Koptiaki. L'identification des ADN nucléaires et mitochondriaux de tous les membres de la famille Romanov mettait un point final, sur le plan biologique, à cette tragique histoire avec, pour épilogue, la canonisation de toute la famille par l'église orthodoxe russe et l'édification sur les hauteurs de lekaterinbourg de l'église Sur-le-Sang-Versé, aujourd'hui lieu de commémorations et de pèlerinages [1]. Pourtant, il manquait encore une pièce à ce puzzle génomique: la nature moléculaire de l'hémophilie du petit tsarévitch. Nombreux sont les historiens qui estiment que la maladie d'Alexis, seul héritier

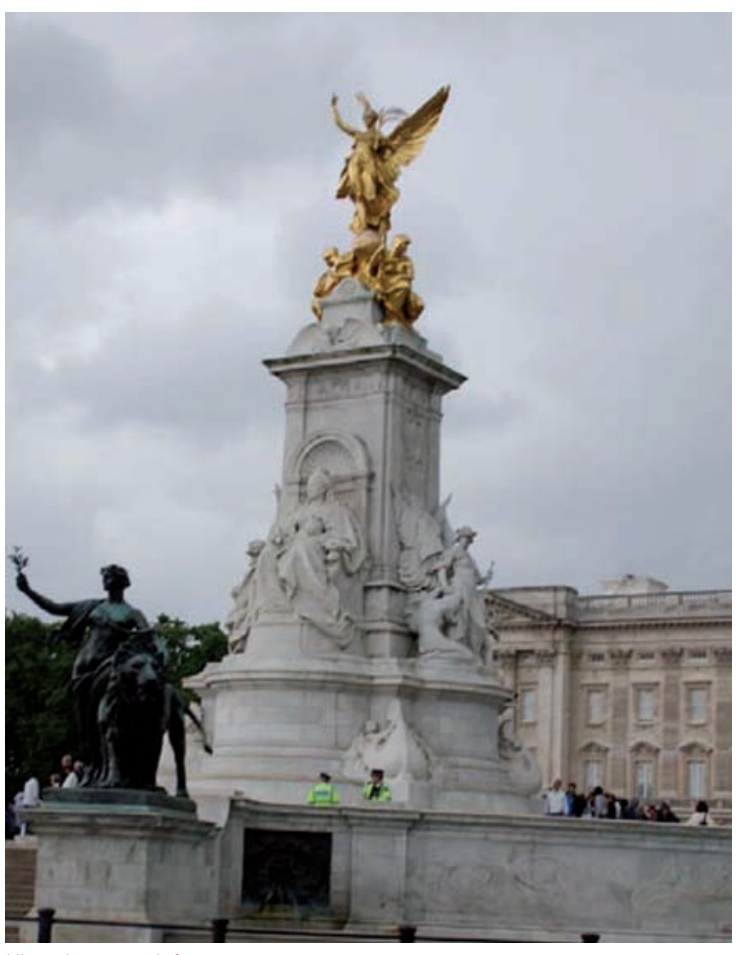

Victoria memorial du trône, a influencé défavorablement la cascade d'évènements qui se succédèrent au cours de la révolution de 1917. Plus sévère que celle de ses cousins espagnols (hémorragie ombilicale à 6 mois, hématomes nombreux au cours des premières années, hémarthrose du genou), elle a suscité chez sa

d'exemple de transmission de l'hémophilie et nous est

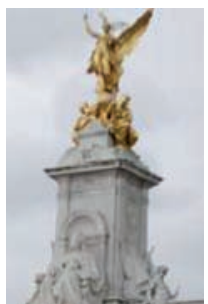

9 , rue Basse,

54330 Clérey-sur-Brénon, France

simsimone.gilgenkrantz@gmail.com

mère, la tsarine Alexandra, une inquiétude d'autant plus vive que celle-ci connaissait parfaitement la gravité de la maladie (responsable de la mort d'un de ses frères, Freidrich, à l'âge de trois ans et d'un de ses neveux, Heinrich, à l'âge de quatre ans). À partir de 1907, la présence de Raspoutine, très mal perçue par l'opinion, a semblé bénéfique sur l'évolution de la maladie. Sa décision de suspendre toute médication, dont l'aspirine, qui, on le sait, aggrave les saignements chez les hémophiles, expliquerait pour certains historiens la relative amélioration. Cette hémophilie, qui avait touché les cours de Hesse, de Russie et d'Espagne jusqu'au début du XXe siècle avait, on le sait, été transmise par la reine Victoria. Du reste, son arbre généalogique a souvent servi

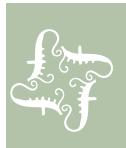
devenu familier, alors que paradoxalement la mutation royale et le type même de l'hémophilie étaient restés inconnus et pour cause: aucun des descendants vivant aujourd'hui n'en étant plus porteur, il était impossible 


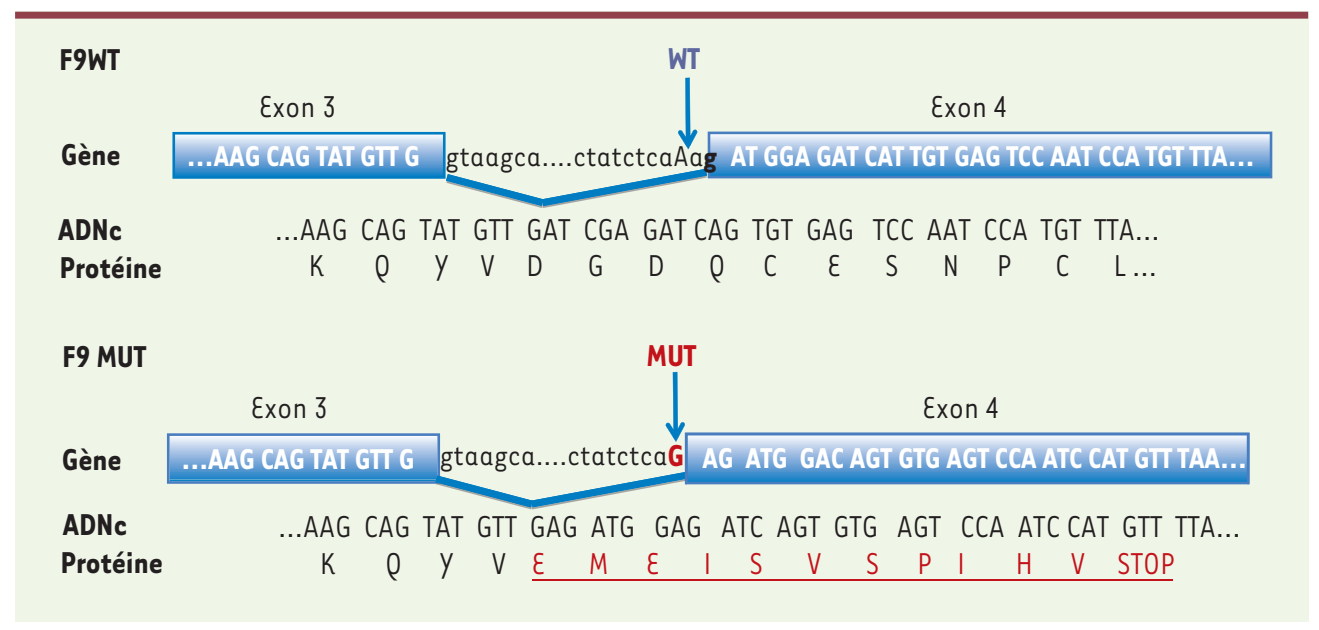

Figure 1. Mutation responsable de l'hémophilie du tsarévitch Alexis. La mutation $\mathrm{G}$->A survient juste en amont de l'exon 4 du gène $F 9$ et est prédite pour créer un nouveau site accepteur d'épissage qui peut entraîner la production d'une protéine tronquée.

de l'identifier sauf à la rechercher dans I'ADN d'Alexis et dans celui de sa mère (petite-fille de la reine Victoria), ce qui vient d'être réalisé. L'étude, effectuée par des généticiens russes et américains, fournit des résultats concluants [2]. Aucune mutation n'a été trouvée dans le gène codant pour le facteur VIII (hémophilie $A$ ). En revanche, et après avoir amplifié l'ADN par PCR multiplex en raison de la faible quantité à disposition, les chercheurs ont trouvé, dans le gène codant pour le facteur IX (F9) (hémophilie B), une substitution $A->G$ localisée dans un intron, à 3 pb de l'exon 4: IVS3-3A-> en position en position 10389 . Or, ce nucléotide est très conservé dans l'évolution et l'étude des transcrits (par piégeage d'exon, combiné au MPS ou massive parallel sequencing) montre que la mutation crée un nouveau site d'épissage préférentiel en IVS3-3 avec glissement du cadre de lecture aboutissant à un codon stop prématuré ( $98 \%$ des transcrits sont générés par ce site et produisent une protéine tronquée) (Figure 1). Ceci explique la gravité de l'hémophilie d'Alexis qui ne devait posséder que 1 \% de fac- teur IX normal. L'analyse moléculaire montre que Alexis est hémizygote pour la mutation tandis que sa mère est hétérozygote. Parmi les quatre filles, une seule était hétérozygote, celle qui est présumée être la princesse Alexandra (Figure 2).

Reste encore une interrogation : avant la reine Victoria, qui a transmis la maladie à trois de ses neuf enfants, aucun cas d'hémophilie n'avait été observé chez ses ascendants. Sans doute s'agit-il d'une mutation de novo chez le père, le prince $\varepsilon$ dward Augustus (il avait 52 ans quand Victoria fut conçue). À moins que, comme le suggèrent certains livres très prisés par les lecteurs de la press people [3], la reine Victoria ait été une enfant illégitime (il semble en effet que sa mère, la princesse Viktoria de Saxe Cobourg Saalfeld ait eu une relation avec son secrétaire, Sir Conroy).

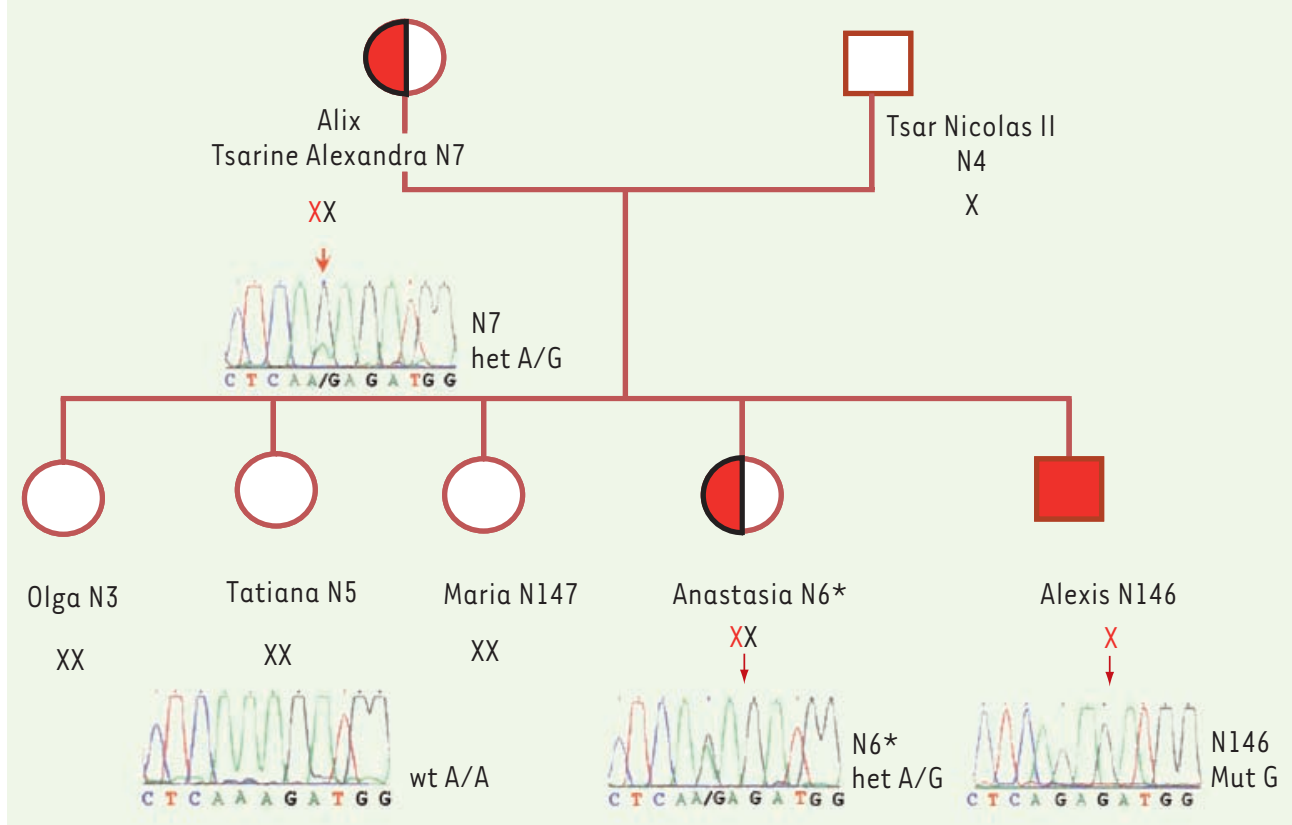

Contrairement à ce qu'auraient pu supposer ces mêmes lecteurs, la «mutation royale »'est pas unique au monde. Elle avait déjà été observée dans deux cas d'hémophilie $B$ sévère, aux ÉtatsUnis dans une famille originaire de l'Europe du Nord-Ouest [4, 5], et plus récemment dans une famille espagnole [6]. Dans les deux cas il s'agit d'une mutation de novo.

Figure 2. Transmission de la mutation dans l'intron 3 (IV S3-3A $\rightarrow G$ ) du gène F9 dans la famille Romanov. 
Il est donc inutile de rechercher un effet fondateur qui relierait ces deux familles roturières à la famille royale d'Angleterre.

Du point de vue scientifique, la découverte de nouveaux cas d'hémophilie B par une mutation sur un site d'épissage déjà rapportée ne mériterait pas publication. Mais ce travail, en utilisant les techniques de génomique actuelles qui remportent des succès sur des ADN encore bien plus anciens [7], a eu le mérite d'obtenir des résultats à partir de corps partiellement détruits par le feu et l'acide sulfurique. Et les lecteurs sont toujours friands, même s'ils s'en défendent, de ces retours anecdotiques sur le passé. $\diamond$

Genomics of the Royal disease

\section{TIRÉS À PART}

S. Gilgenkrantz
CONFLIT D'INTÉRÊTS

L'auteur déclare n'avoir aucun conflit d'intérêts concernant les données publiées dans cet article.

\section{RÉFÉRENCES}

1. Gilgenkrantz S. Le massacre des Romanov : épilogue génomique. Med Sci (Paris) $2009 ; 25: 637-40$.

2. Rogaev $\varepsilon 1$, Grigorenko AP, Faskhutdinova G, et al. Genotype analysis identifies the cause of the Royal disease. Science 2009; 326: 817-23.

3. Mitterrand F. Les aigles foudroyés. Paris: Robert Laffont, $334 \mathrm{p}$.

4. Ketterling RP, Vielhaber $\varepsilon$, Botterma CDK, et al. Germ-line origins of mutation in families with hemophilia $B$ : the sex ratio varies with the type of mutation. Am J Hum Genet 199352 : 152-66.

5. Gianelli F, Green PM, High KA, et al. Haemophilia B: database of point mutations and short additions and deletions. Nucleic Acids Res 1993; $21: 3075-87$.

6. Vidal F, Farssac $\varepsilon$, Altisent $C$, et al. Factor IX gene sequencing by a simple and sensitive 15 -hour procedure for haemophilia $B$ diagnosis: identification of two novel mutations. BrJ Haematol 2000; 111: 549-51.

7. Orlando L, Hänni C. Du nouveau pour l'ADN ancien. Med Sci (Paris) 2000; $16: \mid-X V I$.

\section{Un événement : le nouveau volume indispensable de la prestigieuse collection " Pédiatrie "}

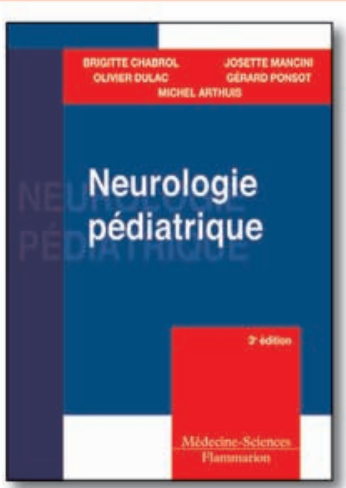

A paraitre le 03/03/2010

1012 pages - 360 figures - 150 tableaux ISBN : 978-2-2570-0030-9 Prix Public TTC : $109 €$

\section{NEUROLOGIE PÉDIATRIQUE - $3^{\mathrm{e}}$ éd.}

\section{Brigitte Chabrol, Gérard Ponsot, Olivier Dulac, Josette Mancini, Michel Arthuis}

Cette $3^{\mathrm{e}}$ édition, totalement réécrite, expose en 1012 pages, 360 figures et 150 tableaux tout ce qu'il convient de savoir en neurologie infantile, en intégrant les progrès fulgurants effectués dans la discipline.

- Un nouveau chapitre très complet porte sur le diagnostic anténatal des pathologies neurologiques, et le chapitre consacré à l'épilepsie a bénéficié d'une refonte complète.

- Les déficiences et handicaps neurologiques sont analysés du point de vue de leurs conséquences médicales, mais également éducatives.

- La place de la pédopsychiatrie dans les maladies neurologiques a été considérablement développée.

\section{Au total, le traité le plus complet et le plus à jour dans le domaine de la neurologie} pédiatrique, aussi bien dans la littérature française qu'étrangère.

En vente chez votre libraire spécialisé, par correspondance ou sur notre site www.medecine.lavoisier.fr ou www.eminter.fr Bon de commande à retourner complété à : LAVOISIER SAS - 14, rue de Provigny - 94236 CACHAN Cedex

\begin{tabular}{|l|l|}
\hline Neurologie Pédiatrique $-3^{e}$ éd. $: 109 €$ TTC & Quantité \\
\cline { 2 - 2 }$(+7 €$ de participation aux frais de port par exemplaire) soit $116 €$. & \\
Frais de port offerts* si paiement joint à la commande. & \\
\hline
\end{tabular}

(*France métropolitaine, Suisse, UE. Autres, nous consulter)

Carte bancaire $n^{\circ}$ :

Les 3 derniers chiffres situés au dos de votre carte bancaire :

Fonction / spécialité : ..................... Adresse :

Code postal :......... Ville : . .

Ces renseignements pourront figurer sur un fichier informatique. Conformément à la loi Informatique \& Libertés du 6 janvier 1978 ,

vous bénéficiez d'un droit d'accès et de rectification aux données vous concernant.
Je joins mon règlement à la commande :

Montant total de : $€$

Chèque bancaire ou postal payable en France à l'ordre de : LAVOISIER SAS (Une facture acquittée sera jointe au colis)

Date d'expiration :

Nom / Prénom :

Tél. : . ........................... E-

$\ldots \ldots \ldots \ldots \ldots \ldots \ldots \ldots \ldots \ldots \ldots \ldots$

Date et signature obligatoire : 


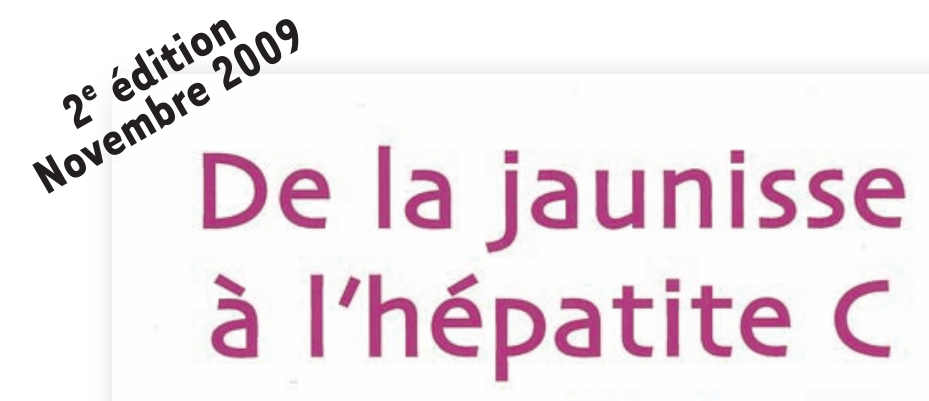

5000 ans d'histoire

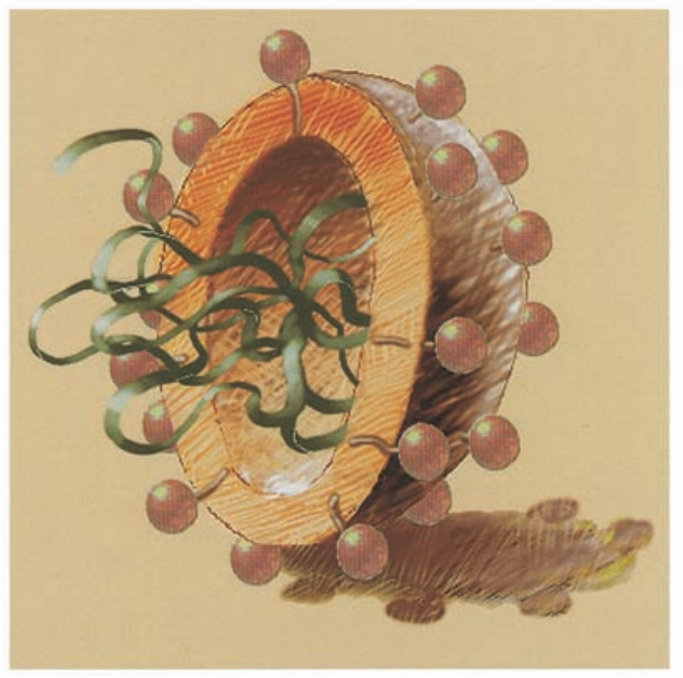

$2^{\mathrm{e}}$ édition mise à jour

Jean-Louis Payen a jaunisse est un symptôme facilement identifiable ; il paraissait bien naturel que l'homme, confronté à une modification de la couleur de ses yeux et de sa peau ait de tous temps recherché les causes de cette transformation.

II n'est donc pas surprenant que le premier traité de médecine, écrit 3000 ans avant J.C. par un médecin sumérien, décrive déjà la jaunisse. À chaque époque de l'histoire de la médecine, les praticiens, influencés par les concepts médicaux de leur temps, attribuèrent une ou plusieurs explications particulières à ce symptôme. Ainsi, du démon Ahhâzu des Sumériens à la sophistication des biotechnologies qui permirent la découverte du virus de l'hépatite $C$, le lecteur cheminera sur une période de 5000 ans au travers des différents continents.

Ici encore, I'histoire se révèle une formidable source de réflexion : le foie souvent impliqué dans l'apparition des jaunisses est-il le siège de l'âme?

Les expérimentations humaines chez des volontaires ou chez des enfants handicapés mentaux étaient-elles justifiées pour permettre la découverte des virus des hépatites?

Le formidable développement de la transfusion sanguine, des vaccinations, mais aussi de la toxicomanie explique-t-il les épidémies d'hépatites du XX' siècle?

Autant de questions qui sont abordées dans ce livre passionnant et accessible à tous.

ISBN : 978-2-8425-4136-1 128 pages

Tél. : 0155641393 - Fax : 0155641394 - ع-mail : edk@edk.fr

NOM : Prénom :

Adresse :

Code postal : Ville :

Pays :

Fonction :

Je souhaite recevoir l'ouvrage

De la jaunisse à l'hépatite $C: 12 €+3 €$ de port $=15 € \Pi C$

en . exemplaire, soit un total de $€$

$\square$ Par chèque, à l'ordre de EDK

$\square$ Par carte bancaire :

Visa $\square$ Eurocard/Mastercard

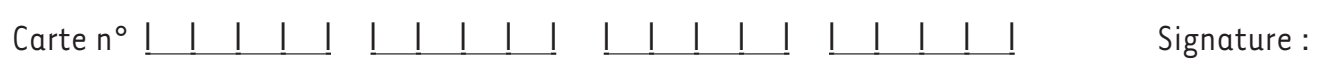

$N^{\circ}$ de contrôle au dos de la carte: 1 । ।

Date d'expiration : 